\title{
UAS FLIGHT TEST FOR SAFETY AND FOR EFFICIENCY
}

\author{
Seamus M. McGovern, U.S. DOT National Transportation Systems Center, Cambridge, Massachusetts
}

\begin{abstract}
Manned aircraft that operate in the National Airspace System (NAS) typically undergo certification flight test to ensure they meet a prescribed level of safety-dependent on their category-before they are able to enter service [for example, Federal Aviation Administration (FAA) advisory circular (AC) $25-7 \mathrm{C}$ is the flight-test guide for certification of transport-category airplanes]. With the integration of unmanned aircraft systems (UAS) into the NAS, in the future some type of certification flight test may ultimately be required, however, even lacking such a requirement UAS manufacturers can find value in flight testing UASs using familiar experimental and certification flighttest procedures, the results of which can enhance the safety of the design, the safety of the operation, and/or the efficiency of the operation. In this paper, selected applications of flight-test procedures to the development and operation of UASs are demonstrated using a rotorcraft UAS (a multirotor) and a surrogate for a fixed-wing UAS. The limited, selected flight tests shown here reveal areas for design improvements to potentially enhance safety, provide insights into UAS and manned-aircraft equipage differences, enable potentially safer operational flight profiles, and facilitate moreefficient flight regimes (e.g., optimal flight profiles to maximize a UAS's flight time). These sample studies show promise for the use of conventional flight-test techniques in the design of UAS flight procedures and in the design of the UAS itself.
\end{abstract}

\section{Aircraft Certification and Associated UAS Test Options}

A wide variety of tests exist for evaluating aircraft [1] many of which can be similarly applied to UAS. These include: regulatory agency certification testing, maintenance flight testing, pilot examinations, pilot operating handbook (POH) comparisons, and experimental flight testing. Other industries also have a variety of ways to evaluate their vehicles, for example, the automotive industry not only makes use of various industry standard tests (e.g., SAE International technical standards and recommended practices) but also takes advantage of competitive racing in order to evaluate new technologies and materials (as it turns out, this may have applicability to UASs as well with the advent of several organizations and sanctioning bodies including the European Rotor Sports Association and the Drone Racing League). Other aviation-related tests and test formats not discussed here include the typically Department of Defense-focused developmental test and evaluation; operational test and evaluation; and research, development, test, and evaluation structures.

In terms of flight test, the military services have their own criteria for evaluating their various aircraft. These requirements are often bound by contractual agreements between the service and the vendor, however these agreements often default to FAA norms.

Maintenance flight testing (also known as or related to production, acceptance, or functional checks) is performed for new aircraft acceptance, post-maintenance performance confirmation, or for in-flight malfunction diagnosis. Maintenance test flights are designed to determine whether the airframe, power plant, accessories, and other items of equipment are functioning in accordance with predetermined standards while subjected to the intended operating environment. Military maintenance test flights are performed after 100-hour inspections and after replacement or removal and reinstallation of any flight controls or surfaces, engines, engine controls, or gearboxes [2]. They may also be performed in some form to diagnose a performance discrepancy noted by an operational pilot or for data collection. Guidelines also exist for commercial-aircraft functional-check flying [3].

Operational flight maneuvers can be used for evaluation of an aircraft for use in its intended purpose (e.g., flying instrument approaches, shipboard interface, etc.). As such, the types of evaluations performed on pilots can be used in providing a test sequence and format for similarly used UASs. Examples of typical pilot evaluations (also known as examinations or check rides) include 
training syllabus flights, annual pilot proficiency evaluations (normal procedures and emergency training), tactical checks (tactical or special procedures), and instrument checks (pertaining to instrument flight rules or IFR). Evaluation using operational flight procedures can be augmented with the Cooper-Harper handling-qualities scale.

Various regulatory agencies are involved in determination of aircraft standards. These agencies have well-established criteria for certifying various types of aircraft. Some of these agencies include the FAA [4] in the U.S., the European Joint Airworthiness Authority in the European Union, the Civil Aviation Authority (CAA) in the United Kingdom, the CAA in Australia, Transport Canada in Canada, the International Civil Aviation Organization (ICAO), and the Royal Aeronautical Society.

In a certificated aircraft, the $\mathrm{POH}$ typically contains various data, charts, and tables developed from testing. Individual datum and data found in tables typically includes airframe limitations (e.g., aerodynamic limitations such as airspeed, acceleration, altitude, and gross weight limits), bestangle-of-climb airspeed $V_{X}$, best-rate-of-climb airspeed $V_{Y}$, stall speeds $V_{S}$ at differing configurations, and center of gravity (c.g.) limitations. Data found in charts is typically performance-oriented and includes takeoff, climb, range, endurance, descent, landing, mission planning (e.g., turn rate versus airspeed, turn radius versus airspeed, etc.), and emergency operation (e.g., glide). This data is a result of test flying, common to certificated and military aircraft, but often lacking in UAS, especially smaller UAS.

Experimental (also known as engineering, developmental, or certification) test flights are conducted to determine or demonstrate critical operating characteristics of an aircraft.

As typical $\mathrm{POH}$ data is often missing from UAS manuals and due to the accepted and rigorous nature in the way $\mathrm{POH}$ data is collected, experimentalflight-test procedures are proposed, not necessarily for UAS certification, but at a minimum to determine what a conventional flight-test program could provide to a UAS manufacturer.

\section{Flight Testing}

The numerous tests available in aircraft certification/experimental flight test can be generalized into two categories: performance and handling qualities. Unlike manned aircraft or radiocontrolled (RC) aircraft, the computer system [commonly referred to as the flight controller (FC), flight controller board (FCB), or autopilot] onboard a UAS is constantly providing for the aircraft's static and dynamic stability. In a manned or RC aircraft the airframe primarily provides inherent stability which may be augmented by the pilot; the level of stability is then measured during flight test. As a UAS's computer provides acceptable stability with no effort on the operator's part, stability testing-while potentially beneficial during a UAS's developmentmay not need to be performed on a developed or operational UAS and will not be discussed here (for more information on stability testing, the reader is directed to [5]). The understanding that any UAS that is being flight tested for the purpose of improving the safety of its design or operation, or for the purpose of enhancing the efficiency of its operation, allows for significantly reducing test planning, conduct, and analysis through the elimination of all handlingqualities tests. The level of testing performed has a dramatic effect on the time required to complete any flight-test study as certification testing [5] includes data collection at many test points (e.g., varying weight, c.g., airspeed, etc.) and test-point combinations for each test, as well as extensive data reduction (e.g., to compensate for aircraft weight changes, an imperfect atmosphere, etc.). Thorough UAS testing can also be performed with significantly less effort than is required in a full aircraft certification program and without compromising rigor through the use of limited testing. This may be appropriate as UASs typically possess protected flight envelopes, excursions beyond which are not allowed by the onboard computer. Restrictions on the size of the operating envelope of the UAS would eliminate the requirement for tests to be repeated at, for example, various speeds, altitudes, weights, and c.g.s (though there is nothing that prevents this; these types of tests are commonly referred to as "spot checks"). 


\section{Flight-Test Procedures}

Performance experimental test-flight procedures as described by the FAA [6], the Society of Experimental Test Pilots [7], the Society of Flight Test Engineers [8], the National Test Pilot School [5], and Askue [9] were studied for determining how these may enhance UAS safety and/or efficient operation. An overview of some experimental-flighttest procedures is provided, which is then followed by three specific flight-test examples planned for application to a rotary-wing UAS and a fixed-wing UAS surrogate.

\section{Pre-Ground-Run Checks}

Conventional aircraft pre-ground-run checks [10] are performed to help ensure that subsequent test flights are free from major controllability or structural issues and may include control system friction and free play, control system resonance characteristics (especially in rotorcraft, any openloop hydraulic control systems are subject to resonance at various frequencies), and airframe resonance characteristics (again especially important for rotorcraft, the natural frequencies of the airframe would be determined by subjecting it to a shake test to ensure that these frequencies are far enough removed from the designed operating or input frequencies so as not to be an issue prior to flight test).

\section{First Ground Run}

The first ground run is used to evaluate the aircraft subsystems and may include the aircraft controls, engine controls, and aircraft subsystems (e.g., electrical, lubrication, hydraulics, etc.).

\section{High-Speed Taxi (Fixed Wing)}

High-speed taxi procedures typically include: repeated taxis (test for roll reversal up to just below stall speed $V_{S}$ ), partial-power land backs (attain $1.3 V_{S}$, throttle to idle, take off, check roll reversal, land), and full-power land backs (attain $1.3 V_{S}$, take off, check roll reversal, throttle to idle, land). Also, the flight-test climb-out/envelope-starting-point speed is calculated using

$$
V_{O}=1.4 V_{s}
$$

Some level of taxi tests can also be performed on rotary-wing aircraft that are equipped with wheels for landing gear rather than skids.

\section{First Flight (Fixed Wing)}

Using $V_{S}$ (clean, and also for gear down, full flaps), $1.3 V_{S}$, and $V_{O}$ at a given test weight, flight-test procedures include: maneuvering climb to test altitude, maneuvering flight characteristics and stalls (flaps up, gear up), maneuvering flight characteristics and stalls (full flaps, landing gear down), accelerating to $V_{O}$ and descend to a planned altitude (e.g,. 800' above ground level or AGL) in the pattern, landing pattern to missed approach, and landing pattern to touchdown (see Askue [9] for further details and test points). With the exception of a V-N plot of the first flight, no data reduction is required.

\section{First-Hover and First-Forward Flight (Rotary Wing)}

The first-hover flight [10] is used to ascertain that the aircraft control functions (i.e. sensitivity, response, and control ranges) are adequate with an emphasis placed on evaluation of the aircraft's controllability and response during slow flight in all directions and during hovering turns. This includes checking for excessive mechanical control coupling, unexpected cross coupling of response, excessive control free play, or extreme control sensitivities. Slow-flight maneuvers are then performed in order to evaluate handling characteristics in forward, sideward, and rearward flight including control reversals, crossed controls, unusual attitudes or rates, unexpected control forces, improper control coupling, improper engine operation, extreme control sensitivities, tail-rotor masking, etc. Ground resonance characteristics are also evaluated at different c.g.s. First-forward flight is then essentially an extension of hover testing and is accomplished to ensure that the aircraft is acceptably stable and controllable to start the flight-envelope gross-weight and c.g. build-up. Static and dynamic stability, mechanical and aerodynamic coupling, proper control sensitivity and response, and adequate control remaining in all phases of power-on and power-off flight are also investigated. 


\section{Verification of C.G. Limits (Rotary Wing)}

For a UAS, c.g. limits can be especially important if the aircraft is being used for delivery of an external load or if the aircraft can hold articulated devices (e.g., a movable camera). Predicted c.g. limits are verified by ascertaining that the aircraft has adequate longitudinal control power remaining throughout the flight envelope at the upper and lower c.g. limits as well as consideration of high dynamic system stresses, excessive fuselage attitudes, or excessive vibrations [10]. For a rotorcraft, fuselage angular acceleration is measured as a function of longitudinal control displacement (step inputs held until the aircraft's pitch rate has reached a steadystate value) while hovering. Following determination of the aircraft's control power, flights showing that adequate control power is available at the most adverse flight conditions (maximum forward speed for the aft c.g. and maximum rearward speed for the forward c.g.) is then be performed (e.g., military specifications require a minimum of 10 percent of the maximum control power available in a hover to be available at these extreme points of the envelope).

\section{Envelope Expansion ( $V-N$ Diagram, Fixed Wing)}

An airplane's envelope is defined by a plot of the load factor in Gs versus airspeed and is referred to as the $\mathrm{V}-\mathrm{N}$ diagram. The envelope is the area within which the airplane is structurally sound and free of flutter for a given weight, altitude, and configuration.

The curved (second-order) vertical line bordering the left side of the envelope is the accelerated-stall line and depicts the largest amount of lift the wing will develop at a given speed without stalling. It starts on the left at $V_{S}$ and one $G$ and moves right and up until the maneuvering speed $V_{A}$ at the positive load limit factor $N+$ according to

$$
N=\left(\operatorname{KTAS} / V_{S}\right)^{2}
$$

where KTAS represents knots true airspeed and $N$ represents the $G$ load. Exceeding this curve will result in a stall.

The top of the envelope is typically a straight, horizontal line described by $\mathrm{N}^{+} . \mathrm{N}+$ is determined by the structural capability of the wing and tail. It should be $2.5 \mathrm{G}$ for transport category aircraft, $3.8 \mathrm{G}$ for normal category aircraft, $4.4 \mathrm{G}$ for utility, and 6.0 $\mathrm{G}$ for aerobatic category aircraft. If the limit load is exceeded, some structural damage may occur in the form of a permanent deformation due to a single application of a high load (static failure) or fatigue damage due to repeated applications of load (fatigue failure). One-and-one-half the limit load is defined as the ultimate load (3.75, 5.7, 6.6, and $9.0 \mathrm{G}$ respectively). If the ultimate load is exceeded, the aircraft could be expected to suffer static failure.

The rightmost vertical border of the envelope (generally a straight, vertical line) is at the neverexceed airspeed $V_{N E}$ (redline airspeed beyond which the aircraft must not be flown). It is defined as 90 percent of the highest speed demonstrated by test and should be about 15 percent greater than the cruise airspeed $V_{C}$. Speeds past $1.1 V_{N E}$ will lead to any one of four aeroelastic effects. These include aileron reversal, wing divergence, wing flutter, or shock wave formation (at the critical Mach number $M_{\text {crit }}$ the airflow velocity is just supersonic over the aircraft while it is still subsonic in the free airstream causing a buffet or shaking that can damage the airframe and cause the control surfaces to become ineffective).

\section{Envelope Expansion ( $V-N$ Diagram, Rotary Wing)}

Rotary-wing $\mathrm{V}-\mathrm{N}$ testing differs from fixedwing testing in the generation of the desired load factor by using a step input to the controls (or a combination of a step input in one axis and a rampor delayed-type input in the other). This is because, unlike fixed-wing aircraft, rotorcraft are normally unable to sustain a design $G$ for longer than a fraction of a second due to blade stall, control-stop contact, excessive rotor droop, attainment of maximum servo rates, droop-stop contact, etc.

\section{Other Flight Tests}

The above and the three processes in the following section are just a few of the available flight-test procedures that could be applied to UAS. Askue [9] categorizes the flight-test process into systems' tests, taxi tests, first flight, envelope expansion, handling qualities-stability, handling qualities - control, pitot static system, stall-spin test, performance, and engine cooling. A typical flight-test program may consist of: high-speed taxi, first flight, 
envelope expansion, high angles of attack (stall and turning flight), maximum rate of climb/excess power, maximum glide endurance/thrust required, static stability handling qualities (longitudinal static stability without trim, and longitudinal static stability with trim), dynamic stability handling qualities (longitudinal dynamic stability for phugoid and for short-period response), lateral and directional dynamic stability cross coupling (directional divergence, spiral divergence, and Dutch roll dynamic effects), control (longitudinal, lateral, and directional), and engine-out performance and asymmetric qualities.

\section{Selected UAS Flight-Test Analysis}

\section{Overview}

Flight-test planning for the purposes of this study was restricted to three items: pre-test planning, rotorcraft height-velocity diagram, and determination of maximum-rate-of-climb and best-angle-of-climb speeds. This limited flight-test planning readily revealed several safety and efficiency issues that could be addressed either in design or operation of the two test-article UASs.

\section{Test-Article Description (Rotary Wing)}

The rotary-wing flight-test article was a familiar multirotor (a quadcopter) UAS designed primarily as an aerial camera and marketed towards the hobbyist. Categorized as a small unmanned aircraft system (sUAS), it was selected for its popularity, its representative design, and its availability, having been acquired by the U.S. DOT Volpe Center as part of another research effort.

The test-UAS's remote control has, for a UAS (and to some extent for aircraft-trained pilots), conventional flight controls. Of the two joysticks, the right joystick is controlled with the right hand and in a manner equivalent to a stick-type flight control in a manned aircraft (fore and aft movement pitches the UAS about its lateral axis, right and left movement rolls the UAS about its longitudinal axis; both are limited to 35 degrees by the onboard computer). The left joystick is controlled with the left hand and performs the function of helicopter collective and pedals in a manned aircraft (fore and aft movement equates to increasing and decreasing a collective- though the rotors' pitch are fixed and the increase/decrease equates to engine speed rather than blade pitch-while this is not exactly the same motion as a collective, it is still found to be intuitive as it is familiar in its motion to an aircraft throttle; right and left movement yaws the UAS about its vertical axis and is limited to 150 degrees per second by the onboard computer). UAS performance and status information (as well as the camera view) is displayed to the operator at the remote control using an operator-supplied smart phone or tablet computer affixed to the remote control. The display includes a variety of information as well as telemetry including the GPS-determined speed (listed as horizontal speed and listed in meters per second or $\mathrm{m} / \mathrm{s}$ ) and altitude (in meters or $\mathrm{m}$ ). The transmitter operates at $2.4 \mathrm{GHz}$ (5.8 GHz and $2.4 \mathrm{GHz}$ are typical for UASs with $2.4 \mathrm{GHz}$ providing a longer range at the expense of a lower data rate).

The UAS's airframe stands on a skid-style landing gear, providing both support for the UAS while on the ground and protection for the camera and its gimbal mechanism. The four rotors are equally spaced and laid out at the corners in a square. The rotors are fixed-pitch with thrust changes manifested though changes in the speed of each rotor's individual electric motor. Two rotors spin clockwise, while the other two spin counterclockwise. The airframe does not appear to have any aerodynamic component; i.e., there are no observed or known lift-generating or significant dragreducing features incorporated. Power to the motors is provided by a single 4,480-milliampere-hour (mAh), 15.2-volt (V) battery having a nine-level power-remaining readout built in and also provided to the remote control's display in percent (from zero to 100 ). The maximum speed is rated at $16 \mathrm{~m} / \mathrm{s}$ (as this is from the GPS, it should be noted that this would be a no-wind speed and is equivalent to 31.10 knots) and the maximum rate of climb is rated at 5 $\mathrm{m} / \mathrm{s}$ which converts to 984.25 feet per minute (fpm). The UAS is indicated to weigh 1,280 grams (g) which would convert to 2.82 pounds, though no maximum gross weight is given.

\section{Test-Article Description (Fixed Wing)}

For the purpose of further explaining some flight-test procedures along with a data-reduction process, actual data collection is demonstrated using 
information from a previous study [11] where a sixdegree-of-freedom flight model underwent flight testing as part of a simulator validation effort. The aircraft model used, the U.S. Navy Beechcraft T-34C Turbo Mentor [2], is similar in size, weight, and performance to a General Atomics MQ-1 Predator. The MQ-1 Predator is categorized as a "Tier II" medium-altitude, long-endurance unmanned aircraft system (Tier II MALE UAS); the T-34C is a singleengine turbine, tandem-seat, aerobatic military training aircraft meeting the FAA definitions of both a complex airplane and of a high-performance airplane (the FAA defines a complex airplane as one that has a retractable landing gear, flaps, and controllable propeller and a high-performance aircraft as one with an engine of more than 200 horsepower). This surrogate provides for an analogous fixed-wing flight-test example.

\section{Example UAS Flight Test and Findings-Pre- test Planning Observations (Rotary Wing)}

Flight-test planning involves gathering and review of available aircraft information. Inspecting the UAS's operating manual reveals that there is limited overlap with aviation protocols. Speeds are in meters per second rather than knots (and, as previously mentioned, those speeds are groundspeeds which, while useful, are not normally used in flight test and are not the convention in aviation). Vertical speed is also in meters per second (versus feet per minute). Weight is in grams rather than pounds. There was no performance planning section and information on flight procedures/maneuvers was limited. A restriction that was recognized was that there would be a limitation to the amount of data that could be collected due to the envelope protection built into this and other UASs.

While it does have a barometer that it uses for determining its height (vs. altitude) relative to its takeoff point, another significant restriction was that this UAS, as is common for many smaller UASs, did not have a true pitot-static system. The aircraft does know its speed, but that speed is provided by GPS. Aircraft with pitot-static systems are flown by (and tested using) indicated airspeed (IAS or knots indicated airspeed KIAS). Calibrated airspeed (CAS) corrects indicated airspeed for instrument error present in the pitot-static system (these errors are aircraft type-specific and can be found in aircraft operating manuals). Equivalent airspeed (EAS) is calibrated airspeed corrected for compressibility effects as the air is rammed into the pitot tube (though the effect is generally negligible below 200 knots). True airspeed (TAS) corrects equivalent airspeed for density variations with altitude, and is the actual speed that the aircraft is moving relative to the air. Groundspeed (GS) is TAS adjusted for wind. As such, this UAS is providing the operator with a groundspeed rather than any speed though an air mass which has implications in testing as well as in safe and efficient operation (e.g., the aircraft and/or operator cannot easily know that the aircraft is operating at an airspeed that is particularly safe for a given operation or flying at a preferred, efficient airspeed). Also, as the reported speed is horizontal speed, in many flight regimes it is insensitive to the aircraft's velocity vector as a pitot-static system would be (at least in relatively balanced flight). For example, a climbing aircraft may use pitch to effect the climb so its horizontal speed (i.e., its GPS groundspeed) would be less than its airspeed. This UAS-reported horizontal speed is another consideration both for flight test and for NAS integration. (While not investigated here, the lack of a pitot-static system is common with smaller fixedwing UAS as well; flight is often conducted via pitch attitude.)

Similar to the airspeed concerns, the aircraft's lack of a pitot-static system means that the altitude it could report is not precisely pressure altitude, as is the convention in aviation. This becomes especially important for aircraft that are to be integrated into the NAS as altitude reporting to air traffic control could be off by a significant margin depending upon weather conditions. This could be even more of a safety consideration for UAS that use their pressure altitude as part of a sense-and-avoid system (e.g., via TIS-B), ADS-B, TCAS II, or any system that uses pressure altitude as reported by participating aircraft.

The flight-test planning also revealed a variety of efficiency issues that could be addressed in the operation of this UAS. The first has to do with the height of the skid landing gear and flight operations in ground effect (IGE). When an aircraft flies (including hovering) at an altitude above the ground approximately at or below the aircraft's wingspan/rotor diameter, the ground restricts the size of the wingtip/rotor vortices resulting in lower induced drag. For example, a Bell 206B helicopter 
[12] requires $70 \%$ torque to hover in ground effect (IGE) but $80 \%$ torque to hover out of ground effect (OGE) at 2,800 pounds on a standard day at sea level. With our test UAS, this has obvious efficiency implications for hovering as well as for a takeoff profile. However, studying the fuselage design reveals that this (i.e., IGE operation) is not possible due the large height of the skids; the aircraft is already OGE before it ever takes off.

Other efficiency observations have to do with the fuselage shape. The design appears to be based primarily on structural layout considerations and aesthetics, however some level of performance enhancements-possibly including increased speed and increased inflight operating time-may be possible with design considerations that include lift generation and/or drag reduction. Increased lift and associated flight test may also result in finding that climbs being conducted with some level of airspeed (i.e., horizontal speed) rather than purely vertical, provides the highest and/or most efficient rates of climb. Fairing the camera assembly, or at least the gimbal mechanism, could provide some level of drag reduction, while fairing the vertical portions of the skids and fairing and shaping the horizontal portions could provide additional drag reduction and lift generation (though this would only occur at most in two directions and with the understanding that generating lift also creates drag). Inverting the rotors (i.e., placing the rotors below the structure rather than above) may provide efficiencies in the same way tractor ("puller," starboard-side mounting on U.S.designed helicopters) tail rotors have been supplemented to some degree by pusher tail rotors (port-side mounting on U.S.-designed helicopters) [13], though stability could be reduced as is demonstrated by the well-known pole-cart stability problem (also known as the inverted-pendulum problem, as a pendulum is stable when hanging downwards but when inverted it is inherently unstable and must be actively balanced to remain upright).

With the majority of UASs-both fixed-wing and rotary-wing-having fixed-pitch propellers, the wealth of information existing in the flight test of general aviation (GA) aircraft can be applied. Flighttest procedures that evaluate electric motors need to be developed, however much of this test knowledge already exists, having been developed in other industries (e.g., rail-transport locomotives). The same is true for fuel-consumption testing (as well as remaining flight-time calculations, etc.) with consideration of batteries rather than the various fuels used by conventional internal-combustion engines.

\section{Example UAS Flight Test and Findings- Height-Velocity Diagram (Rotary Wing)}

The height-velocity $(\mathrm{H}-\mathrm{V})$ diagram is a pair of curves where the shaded areas should be avoided as the pilot may be unable to complete an autorotation landing (Figure 1) without damage to the aircraft or injury to the crew [14]. For example, in Figure 2 it can be seen that the minimum airspeed for executing a safe landing after an engine failure when operating at 300' AGL is 20 knots.

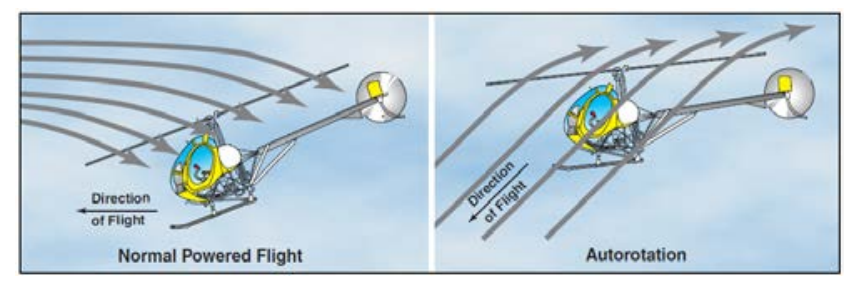

Figure 1. During an autorotation, the upward flow of relative wind permits the main rotor blades to rotate at their normal speed (FAA)

The red-shaded portion in the upper left (referred to here as " $\mathrm{A}$ ”) of the Figure $2 \mathrm{H}-\mathrm{V}$ diagram demonstrates a flight profile where it is unlikely that the pilot could complete an autorotation successfully due to having insufficient airspeed to enter an autorotative profile in time to avoid a crash. The redshaded area on the lower right (referred to here as " $\mathrm{B}$ ") is dangerous due to the high airspeed and proximity to the ground resulting in dramatically reduced reaction time for the pilot in the case of mechanical failure or other in-flight emergency (this shaded area at the lower right is typically not portrayed in $\mathrm{H}-\mathrm{V}$ curves for multi-engine helicopters capable of safely hovering and flying after a single engine failure). 


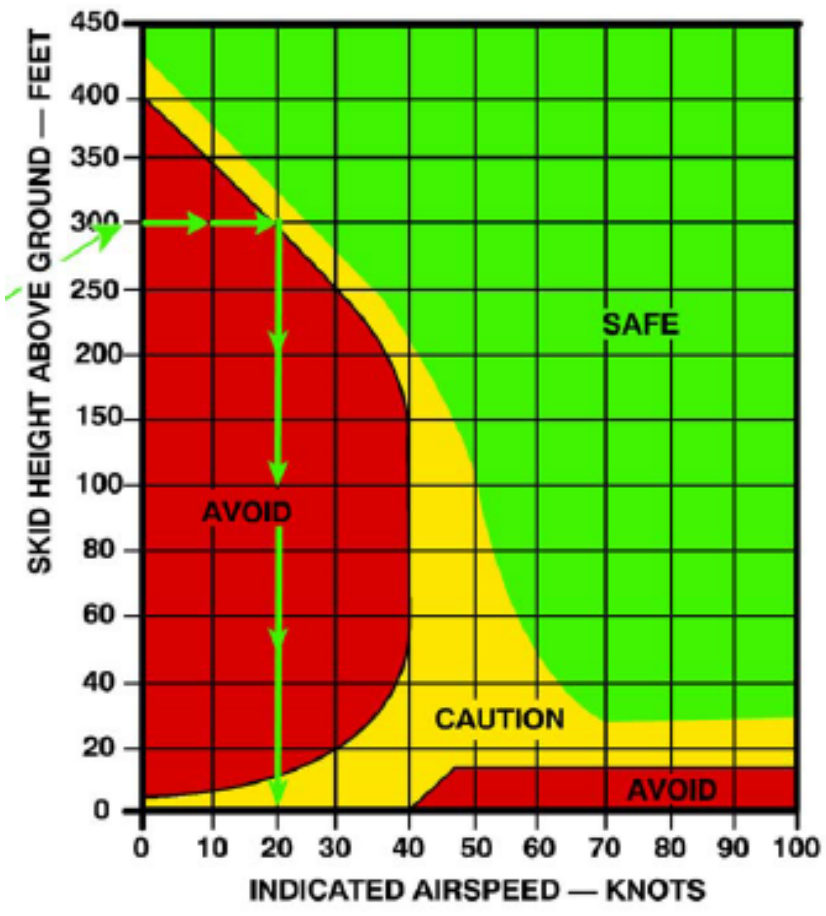

Figure 2. Example of a rotary-wing height-velocity diagram (U.S. Navy)

In terms of developing this curve using flight test, a minimum of three points are required to define area $\mathrm{A}$ and at least two points are required to define area $\mathrm{B}$. In defining area $\mathrm{A}$, testing normally begins at the low-hover point and proceeds up the curve. Aircraft gross weights during testing are usually normal mission weights, while the c.g. location is usually that which will create the most adverse operating condition. Beginning at the zero-airspeed, low-altitude point, an engine failure is simulated and, after a one-second delay, the collective is reduced a sufficient amount to maintain a constant predetermined rotor speed, which is then maintained until the aircraft has descended to an altitude where up-collective application is required to cushion the landing. This maneuver is then repeated at successively higher altitudes until a limitation such as high landing-gear loads or low rotor speed at touchdown is reached. The shape of the diagram around the knee of curve $\mathrm{A}$ is determined in a slightly different manner, where the aircraft starts at the same altitude during successive trial runs while progressively reducing airspeed on each attempt until a minimum airspeed is reached where the same limiting conditions (i.e., low rotor speed or high gear loads) are reached. Several altitude levels at relatively close intervals can then be used to precisely determine the shape of the knee of the curve. (An alternate approach is to hold airspeed constant while reducing altitude; in this approach several airspeeds at close intervals can be used to determine the shape of the knee of the curve.) Determination of the high hover point proceeds in a similar manner, gradually stepping down in altitude from the high starting point to the lowest acceptable test point. After a simulated engine failure, the helicopter is abruptly rotated to a nose-down attitude with the degree of nose-low attitude being proportional to various factors such as the magnitude of the power deficiency, the amount of rotor inertia available, and the altitude at the time of the simulated engine failure. This is usually not more than 15 degrees as previous testing has shown that little performance gain is realized with nose-low attitudes more than 15 degrees and that operational pilots are thought to not be willing to lower the nose much over that without practice [10]. For singleengine rotorcraft, area $\mathrm{B}$ is determined in the same manner as the knee of the curve in area A.

One of the main benefits of the $\mathrm{H}-\mathrm{V}$ diagram is that the curves define a preferred (and safe) takeoff profile; specifically, where the diagram can be traversed from zero height and zero airspeed to cruise altitude and airspeed without entering the shaded areas or with minimum exposure to shaded areas. As such, development of the height-velocity diagram during flight test can lead to the design of safe takeoff profiles. While this would seem to be irrelevant, as most operators of this and other multirotors tend to takeoff vertically with no horizontal component, understanding the $\mathrm{H}-\mathrm{V}$ diagram development and the UAS under study provided several safety insights. The first is the previously seen issue of speed being reported as groundspeed, not the speed of the aircraft through the air mass and varying with air density, limiting the ability to develop an $\mathrm{H}-\mathrm{V}$ diagram. It is also seen that an $\mathrm{H}-\mathrm{V}$ diagram cannot be developed with this UAS (and most multirotors of this size) as, unlike a helicopter, the rotors are directly connected to the motors with no ability to spin freely in one direction in case of an engine failure (to include loss of power supply). Simply adding a sprag clutch (also known as a freewheeling unit) cannot remedy this: Because the UAS's rotors are fixed-pitch, reversing the airflow through them-as occurs during an autorotation in a manned helicopter or an RC helicopter-will not 
have the desired effect when using a sprag clutch in this type of UAS as the rotor (with no ability to change blade pitch) would then reverse direction and so any sprag clutch would, incorrectly, stay engaged with the failed engine, defeating the purpose of the sprag clutch. This insight has safety implications that can then be considered when operating this type of UAS. Alternatively, an understanding of the desired operation and a study of mechanical options yields several design solutions in addition to consideration of changes in operation. These options include familiar centrifugal clutches and electromagnetic clutches, as well as mechanical devices that engage a motor's gears - either through pivoting the motor and its gear or through vertically lifting the motor's gear-when the motor first torques. A centrifugal clutch is a purely mechanical device that uses centrifugal force to progressively throw brake-shoeshaped clutch pads outwards with increasing rotational speed, increasing the connection between two concentric shafts (with the driving shaft nested inside the driven shaft) as rotational speed increases and disengaging with enough of a speed decrease. Electromagnetic clutches are electrical and mechanical devices where, when current flows, an electromagnet produces a magnetic field which engages the clutch; when the current is lost, the magnetic field collapses and the clutch disengages. Including this design would require no specialized switching as the motor is electrical and the clutch's power could be wired in series with the motor's power, automatically disengaging with either a loss of power or a loss of current draw due to a seized motor bearing. While any of these devices add some degree of weight, cost, and complexity-and have the potential of failing themselves - they are not new innovations and are readily available in a variety of sizes. However, even with this ability to disengage from a failed motor, a UAS with fixed-pitch blades could still not autorotate, though this freewheeling capability may reduce-depending on the drag from the freewheeling rotors - the descent rate in the case of a complete power failure (this would obviously necessitate some other solution, such as a ballistic parachute, in the case of passenger-carrying drones). It may also allow for the aircraft to descend in a level attitude, rather than tumble according to the aerodynamic forces acting on the falling airframe. While a reduction in the descent rate and/or a level descent may increase safety in the case of a total power failure, testing could indicate if this is the case and quantify the extent of the benefit to allow for comparison with the tradeoff of increased cost and weight.

Without a redesign of the test-article UAS investigated in this paper, an autorotation is not possible so, in the case of a total power loss, there is no ability to safely glide the aircraft or have a reduced rate of descent. As such, the safest takeoff profile may be the familiar multirotor takeoff profile where the operator directs the aircraft straight up to its operating altitude. This profile makes for a relatively predictable crash location in the case of a total power loss (i.e., straight down to its launch site-which could be expected to have been clear of people and objects during the launch-rather than following a parabolic arc away from that area) and with the lack of horizontal speed effectively reducing the aircraft's kinetic energy during any crash sequence.

However, the most efficient takeoff profile may be very much counter to the typical, purely vertical takeoff profile exhibited by most users. Staying in ground effect (noted earlier as to not be possible with this particular UAS) until attaining best rate of climb airspeed $V_{Y}$ or at least reaching effective translational lift (ETL; see Figure 3 [15]) should provide for the aircraft reaching its desired operational altitude with the minimum amount of energy. ETL occurs while transitioning to forward flight at about 16 to 24 knots. (Rotor blades become more efficient as forward airspeed increases; between 16 and 24 knots the rotor system completely outruns the recirculation of old vortices and begins to work in relatively undisturbed air and, with the flow of air through the rotor system becoming more horizontal, induced flow and induced drag are reduced and the blade angle of attack is effectively increased, which makes the rotor system operate more efficiently which continues with increased airspeed until the best-climb airspeed is reached and total drag is at its lowest point.) Since the test UAS has a maximum attainable speed of just over 31 knots and as tandem-rotor aircraft (e.g., CH47 Chinook) experience ETL, there is no reason to think a multirotor would not as well, however, this is another item that can be verified by flight test. 


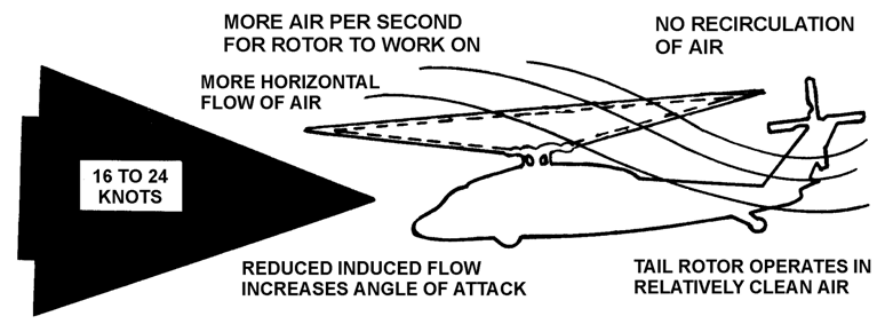

Figure 3. Effective translational lift airflow (U.S. Army)

As the test article UAS used in this paper did not seem to possess any lift-enhancing fuselage shape, both best-rate-of-climb $V_{Y}$ and best-angle-of-climb $V_{X}$ airspeeds may actually occur at ETL, as speeds below this are less efficient and speeds above this may only incur increased parasitic drag. As a result, the most efficient takeoff may be a rapid acceleration IGE to ETL followed by a climb to altitude at ETL.

\section{Example UAS Flight Test and Findings- Maximum Rate of Climb and Best Angle of Climb (Fixed Wing)}

While there are several procedures for obtaining best-rate-of-climb $V_{Y}$ and best-angle-of-climb $V_{X}$ values (a detailed process is described by Kolano [16]), both airspeed values can be readily obtained using a single FAA procedure [6].

Using the T-34C flight model as a surrogate for a similarly sized fixed-wing UAS, best-rate-of-climb speed tests were performed using 3,000' mean sea level (MSL) as the base altitude. A full-throttle climb was initiated 1,000' below the predetermined base altitude with the purpose of stabilizing at the preselected airspeed. These speeds were started at 15 KIAS above the predicted best-rate-of-climb speed and decreased in increments of 5 KIAS down to an airspeed 10 KIAS above $V_{S}$. The resulting target speeds included 115, 110, 105, 100, 95, 90, and 85 KIAS. As the aircraft climbed through the base altitude, a one-minute time check was initiated, at the end of which the altitude was recorded. The aircraft was then descended and the process was repeated at the next speed. The best-angle-of-climb speed tests make use of the same data.

Data reduction consisted of converting the times and altitudes to rates of climb and plotting these versus airspeed using spreadsheet software. The spreadsheet software was then used to fit a second- order polynomial curve to the data. The airspeed that showed the greatest gain in altitude is the aircraft's best-rate-of-climb airspeed $V_{Y}$. Best-angle-of-climb speed is then found by constructing a tangent line from the origin outward to a point on the rate-ofclimb airspeed curve. At this intersection, a line is constructed straight down to the airspeed leg of the chart. The airspeed that the line intersects is the bestangle-of-climb airspeed $V_{X}$.

Test results included a best-rate-of-climb airspeed of $V_{Y}=101$ KIAS (100 KIAS was the value given in the $\mathrm{POH}$ [2]) and a best-angle-of-climb airspeed of $V_{X}=71$ KIAS (75 per the POH) (Figure 4).

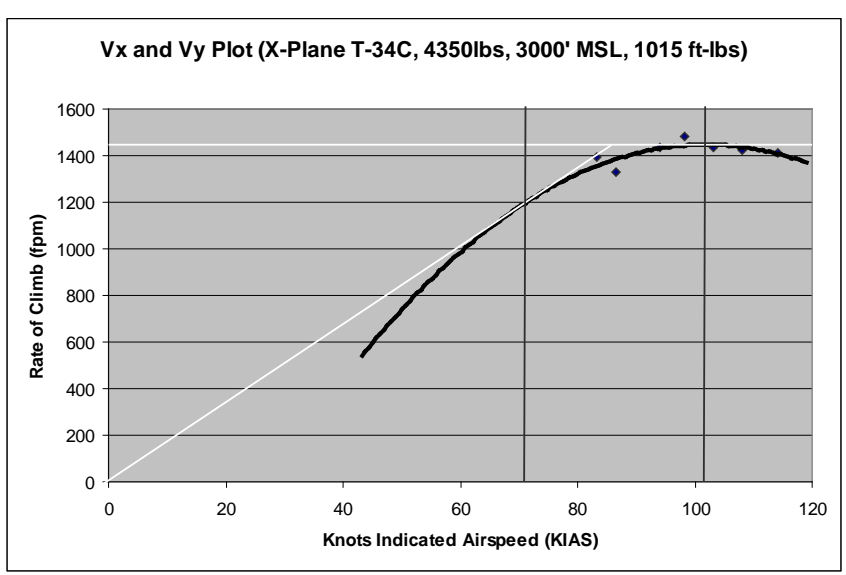

Figure 4. Measured airplane performance curves

Best-rate-of-climb and best-angle-of-climb speeds can only be accurately obtained by flight test and are equally applicable to fixed-wing and rotarywing aircraft. Their uses include minimum rate of descent (e.g., in case of a total power failure), minimum fuel burn rate, maximum obstruction clearance, short-field takeoff, minimum-drag speed, maximum excess power, maximum excess thrust, maximum range, maximum rate of climb, maximum endurance, etc. all of which have value in safe and efficient UAS operations. For example, in terms of safety, $V_{X}$ should be attained by the UAS to avoid an obstacle-to potentially include another aircraft. In terms of efficiency, $V_{Y}$ should be flown to maximize the utility of the UAS before needing to be recharged or refueled. 


\section{Follow-on Efforts}

Follow-on efforts could include design and completion of a full flight-test program on a selected UAS to quantify performance as well as to formulate preferred flight profiles and measure their performance improvements and, where able, their safety improvements.

\section{Summary}

In this paper, a process based in aircraft certification flight test was proposed for quantitatively contributing to and influencing the design and the safe and efficient operation of both fixed-wing and rotary-wing UAS. Three portions of a flight-test program were used to demonstrate safety and efficiency benefits using a popular small multirotor and a surrogate for a larger fixed-wing UAS. The flight-test planning readily revealed several safety issues that could be addressed either in the design or the operation of the UAS. The process demonstrated the objective, rigorous, and qualitative nature of the flight-test evaluation methodology. Even when not required by regulation, flight-test procedures implemented by manufacturers would seem to benefit the design and operation of UASs.

\section{References}

[1] McGovern, S., "Categories for Classification of Aircraft Flight Model Validation," Proceedings of the IEEE/AIAA 26th Digital Avionics Systems Conference, Dallas, TX, October 21-25, CD-ROM, 2007.

[2] U.S. Navy, NATOPS Flight Manual Navy Model T-34C Aircraft, Change 1, Chief of Naval Operations, Washington, D.C., 1984.

[3] Flight Safety Foundation, Functional Check Flight Compendium, Flight Safety Foundation, Alexandria, VA, 2012.

[4] Federal Aviation Administration, Flight Test Guide for Certification of Transport Category Airplanes, Advisory Circular 25-7A, U.S. Government Printing Office, Washington, D.C., 1998.

[5] National Test Pilot School, Introduction to Performance and Flying Qualities Flight Testing,
Second Edition, National Test Pilot School, Mojave, CA, 1995.

[6] Federal Aviation Administration, AmateurBuilt Aircraft and Ultralight Flight Testing Handbook, Advisory Circular 90-89A, U.S. Government Printing Office, Washington, D.C., 1995.

[7] Society of Experimental Test Pilots, Pilot's Handbook for Critical and Exploratory Flight Testing, 2003 Centennial of Flight Edition, Society of Experimental Test Pilots, Lancaster, CA, 2003.

[8] Gardner, L., Painter, D., and Weaver, H. (eds), Society of Flight Test Engineers Reference Handbook, Second Edition, Society of Flight Test Engineers, Lancaster, CA, 2007.

[9] Askue, V., Flight Testing Homebuilt Aircraft, Iowa State University Press, Ames, IA, 1992.

[10] Decker, R., "Rotary Wing First Flight and Envelope Expansion,” Ch. 6, Pilot's Handbook for Critical and Exploratory Flight Testing, Society of Experimental Test Pilots, Lancaster, CA, 1972.

[11] McGovern, S., "Simulator Validation Results and Proposed Reporting Format from Flight Testing a Software Model of a Complex, High-Performance Airplane," Proceedings of the ASME International Mechanical Engineering Congress and Exposition, Boston, MA, October 31-November 6, CD-ROM, 2008.

[12] U.S. Navy, NATOPS Flight Manual Navy Model TH-57B/C Helicopter, NAVAIR 01-H57BC1, Interim Change 17, Commander Naval Air Systems Command (PMA-299), Patuxent River, MD, January 15, 2010 (August 27, 2010).

[13] Wiesner, W., Tail Rotor Design Guide, AD775 391, Boeing Vertol Company, Philadelphia, PA, January 1974.

[14] Federal Aviation Administration, Helicopter Flying Handbook, FAA-H-8083-21A, United States Department of Transportation, Federal Aviation Administration, Airman Testing Standards Branch, AFS-630, Oklahoma City, OK, 2012.

[15] U.S. Army, Fundamentals of Flight, FM 304.4, Headquarters, Department of the Army, Washington, D.C., December 2016. 
[16] Kolano, E., “How to Test Your Airplane’s Best Climb Rate and Vy," Flight Training, Vol. 10, No. 11, pp. 20-27, 1998.

\section{Disclaimer}

Any opinions, findings, and conclusions or recommendations expressed in this paper are those of the author.

\section{Integrated Communications Navigation and Surveillance (ICNS) Conference April 18-20, 2017}

